\title{
Region-of-interest reconstruction from noisy projections using fractal models and Wiener filtering*
}

\author{
AMIT K ROY CHOWDHURY ${ }^{1}$, KAUSHIK BARMAN ${ }^{2}$ and \\ K R RAMAKRISHNAN \\ Department of Electrical Engineering, Indian Institute of Science, Bangalore \\ 560012 , India \\ Present address: " Motorola India Electronics Limited, 33A "The Senate", \\ Ulsoor Road, Bangalore 560002 , India \\ ${ }^{2}$ Silicon Automation Systems (India), 3000, 12th B Main, HAL II Stage, \\ Indiranagar, Bangalore 560008, India \\ e-mail:amitrc@miel.mot.com; kaushikb@sasi.com; krr@ee.iisc.ernet.in
}

\begin{abstract}
In this paper, we present a method for region-of-interest (ROI) tomography using noisy projections. A wavelet decomposition down to the coarsest level is done on the noisy signal. The signal at various levels is estimated using a Wiener filter. By assuming that the projections are $1 / f$ processes, the Wiener filtering reduces to a scalar multiplication. Using the Wiener filter and regularity property of the wavelets, we combine the estimation and localisation of the noisy projections for ROI imaging. Experimental results are shown on Shepp-Logan phantom and actual CT images. The validity of the $1 / f$ model for projections of real life images is also shown.
\end{abstract}

Keywords. Region-of-interest reconstruction; noisy projection; fractal models; Wiener filtering.

\section{Introduction}

In computer-aided tomography, the objective is to reconstruct the cross-section of an object from measurements that are strip integrals of some property of the object. In transmission $\mathrm{X}$-ray tomography, the measurements consist of integrals (projections) of the attenuation coefficient $\mu(x, y)$ of the object along strips which represent the path of the $\mathrm{X}$-rays through the object. A popular technique for image reconstruction is the Filtered Back Projection (FBP) (Kak \& Roberts 1986).

In this work, we are concerned with the problem of reconstructing a portion of the image from noisy projections. The discussions in this paper are restricted to parallel beam

*The names of the authors are in alphabetical order 
tomography. Reconstruction of only a portion of the cross-section being imaged (regionof-interest tomography or local tomography) leads to reduction in the radiation exposure to the patient and savings in computation (compared to FBP of the entire object).

However, the problem of local tomography is complicated by the fact that it is not uniquely solvable in even dimensions (Natterer 1986). Most of medical imaging is conducted in 2-D and the reconstruction formula becomes globally dependent on the lineintegrals of the object. Using wavelets is one method of solving this problem.

The FBP algorithm does not produce satisfactory reconstruction with noisy projections. The work reported here combines the ideas of multiresolution analysis and multiscale estimate of the projections using wavelets. We use a $1 / f$ model for the projections and a Wiener filter for the estimation. It is further shown that this algorithm can be extended to case of ROI tomography.

\section{Preliminaries}

\subsection{Radon transform and its inversion}

The two-dimensional Radon transform, ${ }^{1} \mathbf{f}(r, \theta)$, of a function $f(x, y) \in \mathfrak{R}^{2}$ along a line $L$ parameterized by $(r, \theta)$ such that $r=x \cos \theta+y \sin \theta$ is defined as (Kak \& Roberts 1986)

$$
\mathbf{f}(r, \theta)=\int_{\mathfrak{R}^{2}} f(x, y) \delta(r-x \cos \theta-y \sin \theta) \mathrm{d} x \mathrm{~d} y .
$$

The one-dimensional function $f(r, \theta)$ is termed as the projection of $f(x, y)$ at the angle $\theta$. The back-projection operator denoted as $\mathcal{B}$ is defined as (Kak \& Roberts 1986)

$$
h_{B}(x, y)=\mathcal{B} h(r, \zeta)=\int_{0}^{\pi} h(x \cos \theta+y \sin \theta, \zeta) \mathrm{d} \theta .
$$

Then it can be shown that

$$
f(x, y)=\mathcal{B F}^{-1}[|\omega| \mathcal{F} \mathbf{f}(r, \theta)] .
$$

Equation (3) is the filtered back-projection implementation of the inverse Radon transform. It means that we filter each projection by a $|\omega|$ filter and then backproject the filtered projections.

2.1a Nonlocality of the Radon inversion: The Radon inversion formula can be represented as follows (Olsen 1996):

$$
f(x, y)=\mathcal{R}^{-1} \mathbf{f}(r, \theta)=\mathcal{B H}_{r} \frac{\partial}{\partial r} \mathbf{f}(r, \theta),
$$

where $\mathcal{H}_{r}$ is the Hilbert transform operator defined as

$$
\mathcal{H}_{r} f(r, \theta)=\frac{1}{\pi} f(r, \theta) * \frac{1}{r} .
$$

\footnotetext{
${ }^{1}$ We denote the Radon transform and Fourier transform operators by $\mathcal{R}$ and $\mathcal{F}$ respectively.

$2 \Re$ represents the set of real numbers.
} 
The Hilbert transform imposes a discontinuity on the Fourier transform of any function whose average value is not zero and also discontinuities on the higher derivatives which are not zero at the origin (Olsen 1996). This is because

$$
\mathcal{F H} f(t)=\mathcal{F}\left(f(t) * \frac{1}{\pi t}\right)=\frac{1}{i} \operatorname{sign}(\omega) F(\omega) .
$$

Because of the discontinuities in the frequency domain, there is a spreading of the support of the function in the time domain (Olsen \& DeStefano, 1994). However, this spreading of the function's support will not occur if the function's Fourier transform and the higher derivatives of it are zero at the origin (Olsen \& DeStefano, 1994). If the Fourier transform or higher derivatives of it have zeroes at the origin, it implies that the function has a number of zero moments. Hence functions with arbitrarily high zero moments will have their support unchanged by the Hilbert transform. It is in this context that the wavelet transform is used. Wavelets are usually constructed with many zero moments (Daubechies 1988). Thus local properties of the high resolution components of a wavelet transform remain local after applying the Hilbert transform. It has been shown in (Olsen \& DeStefano, 1994; Delany \& Bresler, 1995) that using wavelets which have several zero moments, the wavelet transformed filtered functions will have essential compact support.

\subsection{Wavelets}

2.2a Continuous wavelet transform of $1-D$ signals: The continuous wavelet transform $W_{\psi}(f)(a, b)$ of a signal $f(t)$ is defined as (Chui 1992):

$$
W_{\psi}(f)(a, b)=\int_{-\infty}^{\infty}|a|^{-1 / 2} f(t) \overline{\psi\left(\frac{t-b}{a}\right)} \mathrm{d} t,
$$

where $a \in \Re^{+}, b \in \Re^{3}$ are the dilation and translation, respectively, of a single wavelet function $\psi(t)$ called the mother wavelet. $\bar{\psi}$ denotes complex conjugation. A very important property for wavelets is the regularity, which means the smoothness of the wavelet function. Regularity $R$ of the mother wavelet $\psi(t)$ is defined as (Aldroubi, 1996):

$$
\int_{-\infty}^{+\infty} t^{r} \psi(t) \mathrm{d} t=0
$$

where $r$ is an integer such that $0<r<R$.

Thus a mother wavelet with regularity $R$ has $R-1$ vanishing moments or $\Psi(\omega)$ (Fourier transform of $\psi(t))$ has a zero of order $R$ at origin, because (Chui 1992):

$$
\int_{-\infty}^{+\infty} t^{r} \psi(t) \mathrm{d} t=\left.(j)^{r} \frac{d^{r} \Psi(\omega)}{\mathrm{d} \omega^{r}}\right|_{\omega=0}=0
$$

It can be shown that a Daubechies' wavelet of length $2 L$ has regularity $R=L$ (Daubechies 1988).

$\overline{39}$ and $\Re^{+}$represent the set of real numbers and the set of positive real numbers respectively 


\subsection{Multiresolution analysis (MRA)}

Given a function $f$, it is possible to obtain the low resolution signal, $f^{(j)}$, and $d^{(j)}$, at a scale $j$ in the following manner (Daubechies 1992):

$$
f^{(j)}(k)=\left(h(.) * f^{(j+1)}(2 k)\right)
$$

and

$$
d^{(j)}(k)=\left(g(.) * d^{(j+1)}(2 k)\right),
$$

where $*$ represents 1 -D convolution. $h$ and $g$ are two filters associated with MRA. The finer scale approximation coefficients $f^{j+1}(l)$ can be synthesized from the next coarser scale approximation and detail coefficients, $f^{(j)}$ and $d^{(j)}$, as

$$
f^{(j+1)}(k)=\sum_{n} \tilde{h}(2 n-k) f^{(j)}(n)+\sum_{n} \tilde{g}(2 n-k) d^{(j)}(n)
$$

where $\tilde{h}$ and $\tilde{g}$ are defined as $\tilde{h}(n)=h(-n)$ and $\tilde{g}(n)=g(-n)$.

\section{$2.41 / f$ processes}

A $1 / f$ process is a nonstationary random process with a power spectral density (PSD)

$$
S(f)=\frac{\sigma^{2}}{|f|^{\gamma}},
$$

where $\sigma$ is a constant and $\gamma$ is known as the spectral exponent.

It can be shown that (Wornell \& Oppenheim 1992)

$$
D=2.5-\frac{\gamma}{2}
$$

where $D$ is known as the fractal dimension. If we choose gamma such that $1<\gamma<3$, then $1<D<2$. Hence fractal dimension of the signal is not an integer, but a real number which lies in $(1,2)$ and the signal is self-similar. This type of signals (1-D random process) are known as fractal signals. Here we concentrate on fractal signals and take $1<\gamma<3$.

\subsection{Statistical properties of discrete wavelet transform coefficients}

From the regularity condition of the mother wavelet, $\Psi(\omega)$, the Fourier transform of $\psi(t)$, has a zero of order $R$ at the origin of the frequency plane. It ensures processing of the signal with mother wavelet essentially removes the low frequency components and we obtain the detail coefficients. These detail coefficients again are wide-sense stationary (WSS) process, even when the signal is non-stationary (Masry 1993). This shows that non-stationarity is concentrated around zero frequency. In our analysis, we have assumed our signal to be stationary. However, in case of any non-stationarity present in the signal, wavelet filtering will effectively remove it. Consequently, processing with only detail signals turns out to be simpler than processing the original signal itself. We have adopted this method in our work. 


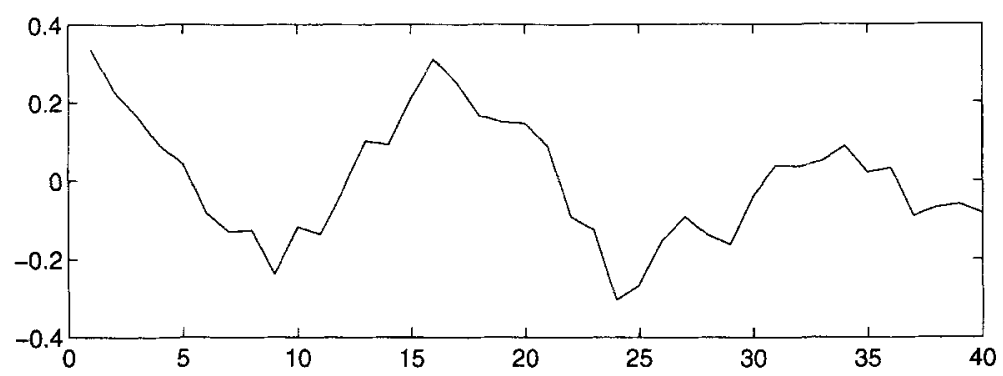

(a)

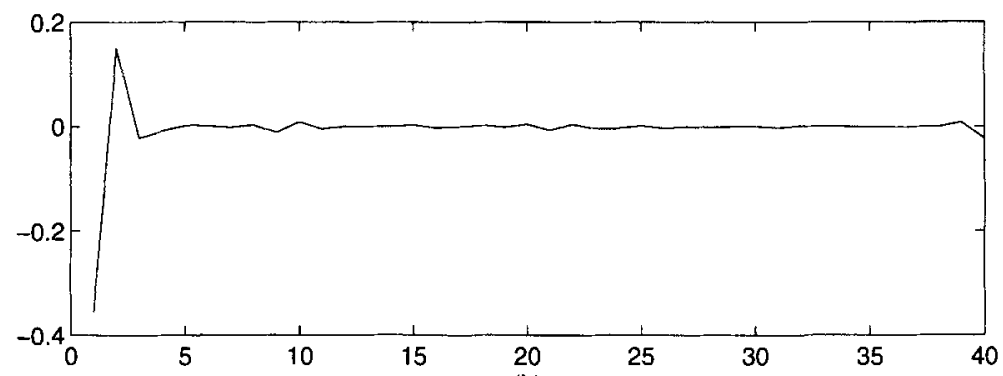

(b)

Figure 1. ACF of highest level detail coefficients with (a) Haar wavelet, (b) with Daubechies' wavelet.

DWT not only makes detail signals WSS but almost decorrelates them also. Correlation structure of detail coefficients can be obtained by calculating autocorrelation at a given scale and over scale to scale. Denoting detail coefficients at scale $m$ (resolution level $2^{m}$ ) as $d_{m}^{k}$, where $m$ and $n$ are the dilation and transiation indicies respectively, it can be shown that (Barman 1997)

$$
E\left[d_{m}^{k} d_{n}^{l}\right] \sim \mathcal{O}\left(\left|2^{m} k-2^{n} l\right|\right)^{(\gamma-R-1)} .
$$

This equation gives the correlation across scales, as well as at a particular scale. Consequently, with higher regularity mother wavelets it is possible to achieve fast decay of correlation coefficients. This property is illustrated in figure 2.5 . Furthermore, it can be shown that the detail coefficients are mutually uncorrelated and their variance decreases geometrically with scale $m$ and at each scale the variance, $\sigma_{2^{m}}$, can be expressed as (Wornell 1993)

$$
\sigma_{2^{m}}^{2}=\sigma^{2} 2^{-\gamma m} .
$$

\section{Wiener filtering}

Here we assume that the original signal itself is a Gaussian process and corrupted by an independent additive white Gaussian noise (WGN). Clearly Wiener filtering gives an optimal estimate of the uncorrupted signal since it minimises the mean square error in the Gaussian case. Instead of doing Wiener filtering directly on the signal itself, we uecompose it into its detail signals at various scales (starting from the highest scale) using wavelet decomposition and do Wiener filtering on these detail signals only. Here we exploit the fact 
that wavelet decomposition whitens the $1 / f$ process. Complexity of estimation process is greatly reduced by adopting this technique (Wornell \& Oppenheim 1992).

Let $r_{n}^{m}, w_{n}^{m}$ and $x_{n}^{m}$ be the detail coefficients of the corrupt signal, additive WGN and the original uncorrupt signal at a scale $m$ (level $2^{m}$ ). Hence, $r_{n}^{m}=x_{n}^{m}+w_{n}^{m}$. We will be doing Wiener filtering at each scale and the problem is to find out a filter with impulse response $h_{n}^{m}$ so that for the input $r_{n}^{m}$ the output will be an optimal estimate of $x_{n}^{m}$. Denoting by $\hat{x}_{n}^{m}$ the optimal estimate of $x_{n}^{m}$ we have,

$$
\hat{x}_{n}^{m}=r_{n}^{m} \star h_{n}^{m}=\sum_{k=-\infty}^{+\infty} r_{n-k}^{m} h_{k}^{m}=\sum_{k=-\infty}^{+\infty} r_{k}^{m} h_{n-k}^{m},
$$

where $\star$ denotes linear convolution.

The optimal filter impulse response $h_{n}^{m}$ at a given scale $m$ is given by

$$
h_{n}^{m}=\frac{\sigma^{2} \beta^{-m}}{\sigma^{2} \beta^{-m}+\sigma_{w}^{2}} \delta[n],
$$

where $\sigma$ and $\beta$ are signal parameters, $\sigma_{w}^{2}$ is the variance of the WGN, $\beta=2^{\gamma}$ and $\sigma$ is defined by the equation $\sigma_{2^{m}}^{2}=\sigma^{2} 2^{-\gamma m}$, where $\sigma_{2^{m}}^{2}$ is the variance of the detail coefficients at resolution $m$.

From the above equation we see that Wiener filter reduces to a scalar multiplication. Here Wiener filtering is done only on detail coefficients because for a $1 / f$ signal most of the energy is concentrated at low frequencies and consequently least affected by the additive white Gaussian noise.

\section{ROI reconstruction}

We now consider the problem of ROI reconstruction when the initial projections are noisy. It is shown that projections at a different angles can be considered as fractal signals, with different values of $\gamma$.

Data Collection: The non-locality property of Radon transform does not permit ROI reconstruction from projections only over that region. It requires line integrals beyond ROI. However, projections can be taken for the entire object (which is to be reconstructed at a coarse resolution) at a low number of angles and the values of the projections for the missing angles interpolated suitably (Olsen \& DeStefano 1994). Over the ROI, projections at an increased number of angles are taken. A comprehensive explanation of the data collection strategy can be found in (Delany \& Bresler 1995; Olsen \& DeStefano 1994).

The 1/f model and filtering: In many applications, the projections will be noisy and a conventional FBP will give poor results. In this algorithm, Wiener filtering is used to remove the noise. The filtering algorithm requires a prior model for the data to be estimated. Analysis of a typical projection data shows that it is a fractal signal. Figure 2 shows the plot of the variance of the detail coefficients at various resolutions of a few 


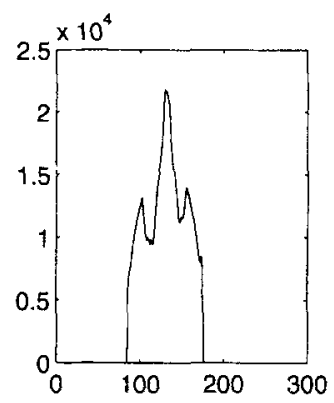

(a)

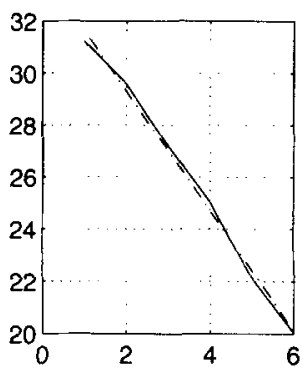

(d)

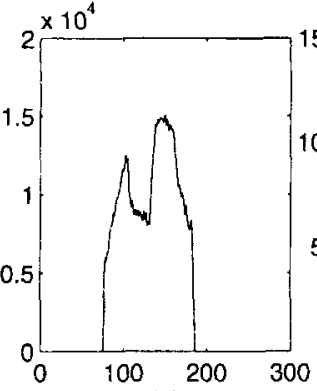

(b)

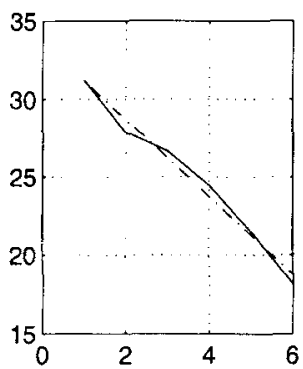

(e)

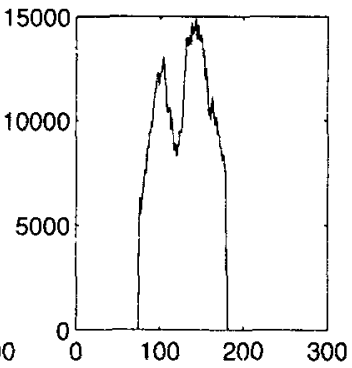

(c)

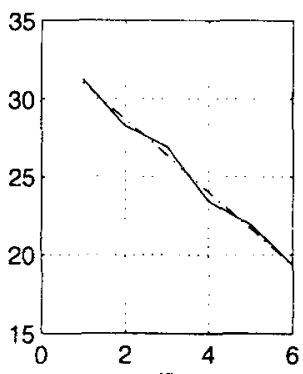

(f)

Figure 2. A few typical projection data and their approximation to the fractal model; actual variance of detail coeficients. against scale:---- straight-line fit.
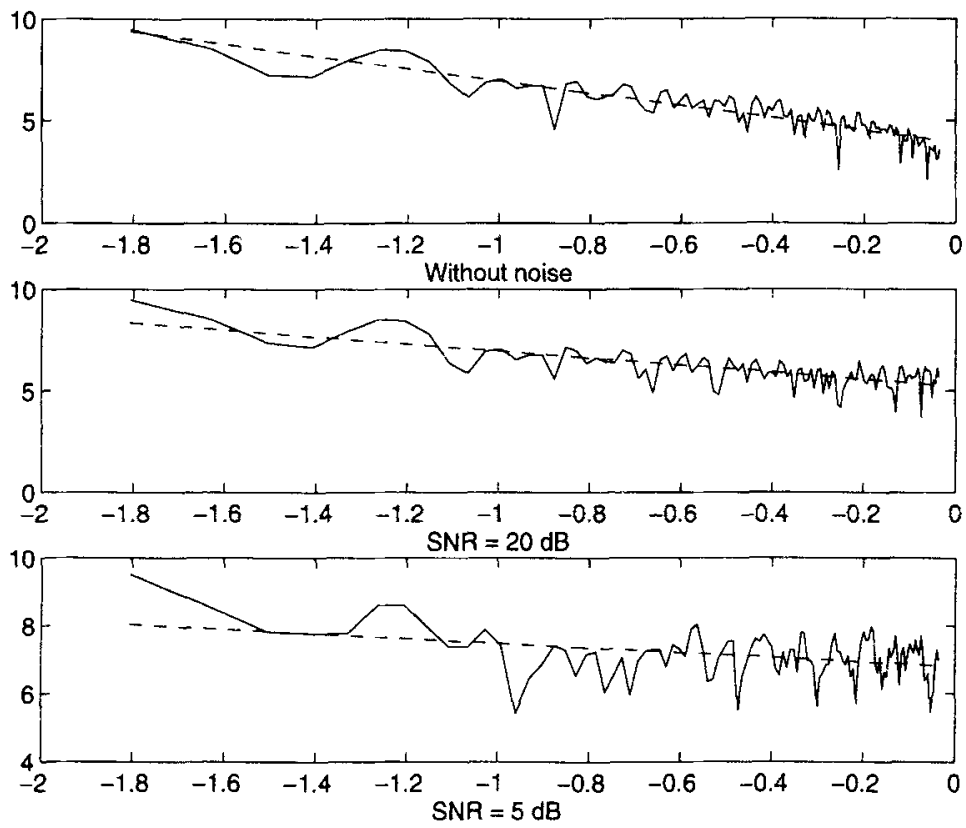

Figure 3. Continuous line shows PSD of a $1 / f$ signal and the dashed line gives a least squares fit. Estimation of fractal dimension becomes poorer with decrease in SNR. 
typical projections against the resolution on a log-log graph. The linear trend in the data in self-evident.

The Wiener filtering process involves decomposition of the signal to the level where the length of the signal corresponds to the filter length. As an example, if a 5th order Daubechies filter is used the signal length at the lowest level will be ten. This may involve padding the signal with suitable number of zeros so that the signal length is equal to filter length $\times 2^{k}$ where $k$ is an integer, denoting the resolution level. It means that if we have a signal of length 256, we zero-pad it upto 320. At level 5, the signal length will be ten, the filter length. The reconstructed signal, after an inverse wavelet transform, is an optimal estimate of the original signal.

Estimating fractal signal parameters: Maximum likelihood (ML) estimation method for fractal signals was introduced by Wornell \& Oppenheim (1992). In this method wavelet decomposition technique is used as a tool and wavelet filter banks not only (almost) decorrelate the $1 / f$ signal but provide a power law variance structure for detail signals also (figure 3 ). Here it is assumed that the original $1 / f$ signal has been corrupted by an additive white Gaussian noise and we estimate fractal dimension from the corrupted signal using Wornell--Oppenheim ML estimation algorithm (Wornell \& Oppenheim 1992).

\subsection{Algorithm for $\mathrm{ROI}$ reconstruction}

Figure 4 outlines the entire filtering and reconstruction process for a simple example. It should be noted that the diagram is simply for the purpose of explanation. The values shown do not reflect the values we used in our simulation. Note that the full exposure projections are sparsely sampled in the angular variable. In the block diagram, there are $N / 2$ full exposure projections and $N / 2$ reduced exposure projections. This effectively means that there are $\mathrm{N}$ projections over the ROI and $N / 2$ away from it. The algorithm is explained in the steps below.

(1) A wavelet decomposition is done on each full exposure projection till signal length is equal to filter length. The detail coefficients are estimated using the Wiener filter. By adding only the detail coefficients corresponding to the projections over the ROI, we obtain the estimate of the projections over the entire object.

(2) A wavelet decomposition is done on the reduced exposure projections. These are at intermediary angles and of smaller length. At the lowest possible resolution, their length is equal to the filter length. The low resolution coefficients of these projections are not processed as they are not localised. Thus before doing a wavelet synthesis on these reduced exposure detail coefficients, we must obtain the low resolution coefficients for these angles.

(3) To obtain the low resolution coefficients for the intermediary angles, we perform an interpolation in the angular variable. The interpolation requires the low resolution data. This is obtained from the synthesis portion of the full exposure projections (see figure 4). The data at the proper scale must be taken. Once we obtain the low resolution coefficients at the intermediate values, we can do the inverse wavelet transform on 
N/2 Full exposure projections of length 40

N/2 Reduced exposure pxojections of length 20

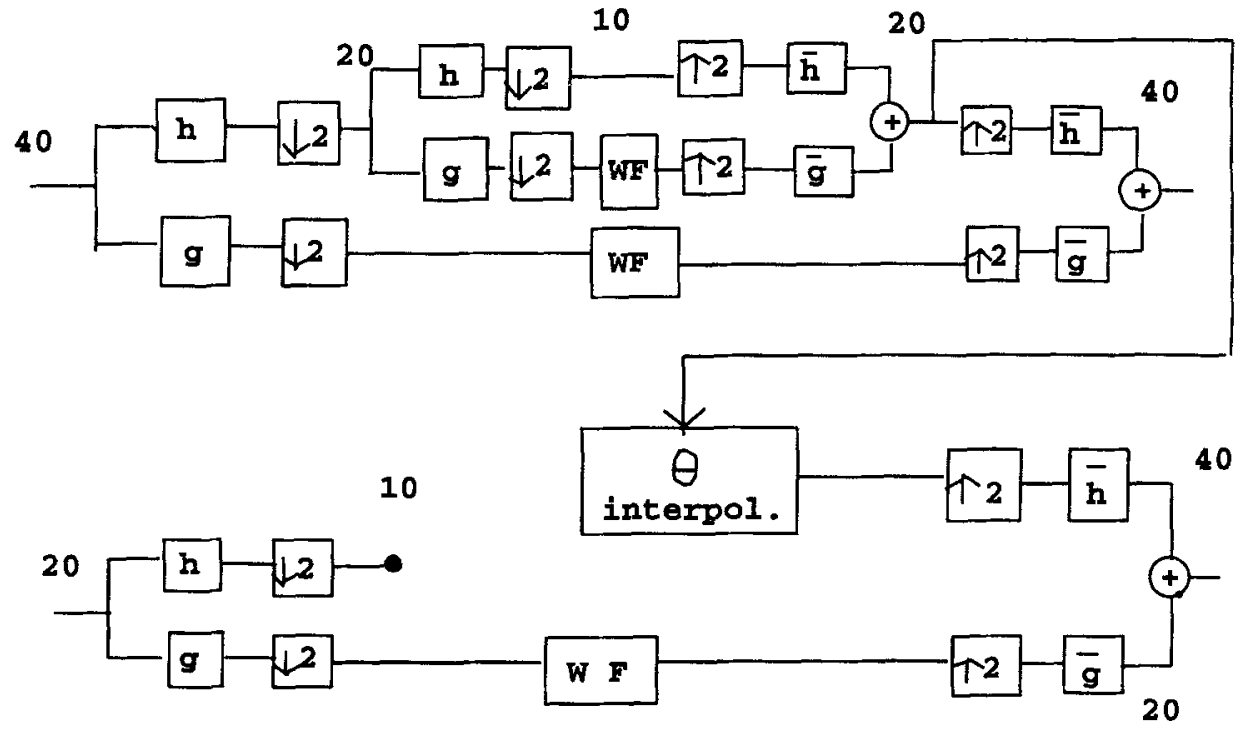

Figure 4. Simple schematic of the algorithm (the values are simply for the purpose of explanation; they do not reflect the values used in simulations), showing the wavelet decomposition, Wiener filtering and wavelet synthesis. Numerals denote signal lengths at various points. $h$ and $g$ are of length 10 .

them, using the detail coefficients available for the ROI. Note that the detail coefficients for a particular resolution will be smaller in length than the low resolution coefficients, as the former is obtained only for the ROI. Thus the addition of the detail coefficients must be only over the ROI.

(4) The projection data for all the angles is now obtained, with the detail information added only to the ROI.

(5) FBP of the result obtained in step (4) results in an object reconstruction which has a high resolution over the $\mathrm{ROI}$ and a low resolution elsewhere.

By stopping the synthesis of wavelet coefficients at a particular scale, we can obtain images at various resolutions. This can also be done sequentially, and the radiologist can view the image at finer and finer resolutions.

\section{Experimental results and comments}

The effectiveness of our algorithm is shown by reconstructing the Shepp-Logan phantom image as specified in standard medical imaging literature (Kak \& Roberts 1986) and actual CT scan images. The image size is $128 \times 128$ in all the cases. The projections are taken 


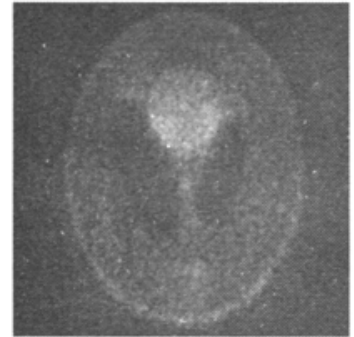

(a)

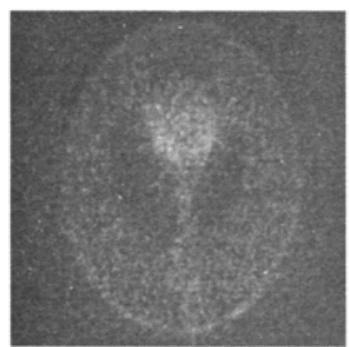

(d)

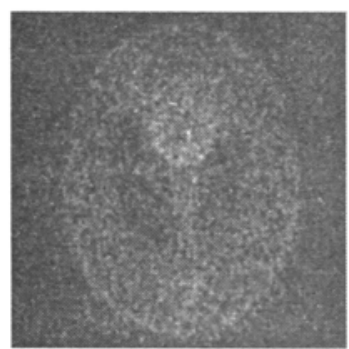

(g)

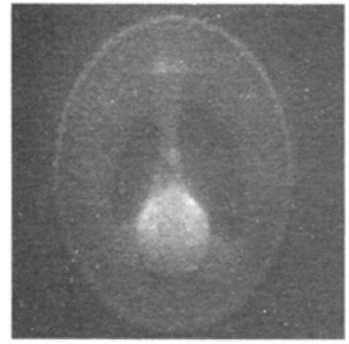

(j)

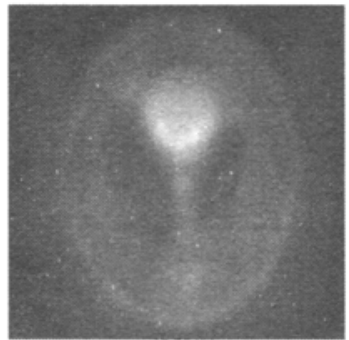

(b)

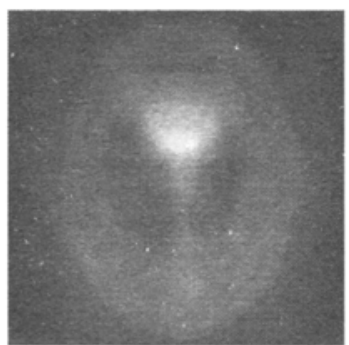

(e)

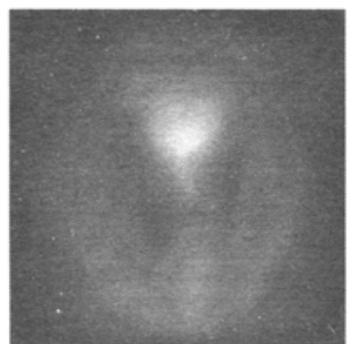

(h)

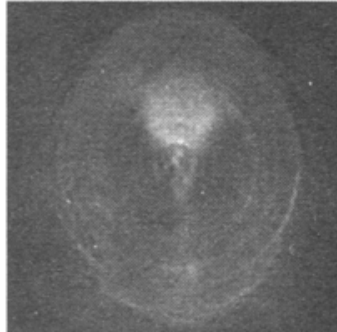

(c)

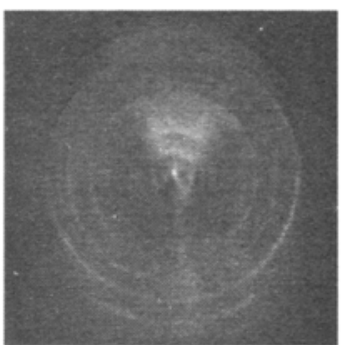

(f)

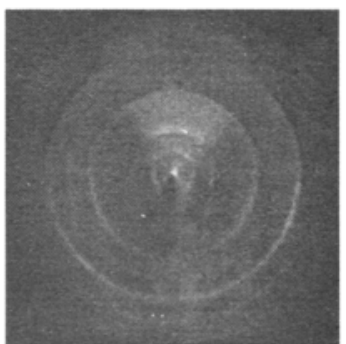

(i)

Figure 5. Noisy and estimated images: (a), (d), (g) - noisy images at 15,10 and $5 \mathrm{db}$ respectively; (b), (e), (h) - images using Daubechies' wavelet for estimation; (c), (f), (i) images using Haar wavelet for estimation. 
at 128 angles for all the images. Over the ROI the number of projections is doubled. Each projection of the phantom image consists of 128 parallel rays while each projection of the actual CT images consist of 193 (approx. $128 \sqrt{2}$ ) rays. The ROI is defined to lie at the centre and approximately covers $1 / 4$ th of the image. The ROI can be shifted to some other part by a change of co-ordinates. We have used Daubechies' wavelets of length 10 ( $h$ is of length 10) (Daubechies 1992).

In figure $5 \mathrm{j}$, the image is reconstructed from the full projection data at 128 angles without any noise. The effect of noise on the data when no estimation techniques are used is shown in figure $5 \mathrm{a}, \mathrm{d}$ and $\mathrm{g}$, for 15,10 and $5 \mathrm{~dB}$. The result of using the filter with the Daubechies wavelet is shown in figure $5 b$, e and $h$. It is apparent that noise has been effectively removed. The smoothing of the edges can be explained by looking at the corresponding projection. A typical projection and the output after filtering is shown in figure 6 . The high frequency details of the projections are removed as the filtering is unable to differentiate the high frequency signal from the noise. For purposes of comparison, we also reconstructed using the Haar basis. The presence of circular rings can be accounted by studying the corresponding reconstructed projection. The estimated projection contains sharp edges. On back-projection, the sharp edges lead to rings in the image. The sharp edges in the estimation using the Haar wavelet is because of several factors. The estimation process assumes that the wavelet coefficients are decorrelated from scale to scale. Figure 1 shows the autocorrelation function with both the Haar and Daubechies' wavelets. The decorrelation in the second case is greater than in the first. Thus our assumption is more correctly adhered to in the second case. As has been shown before, the
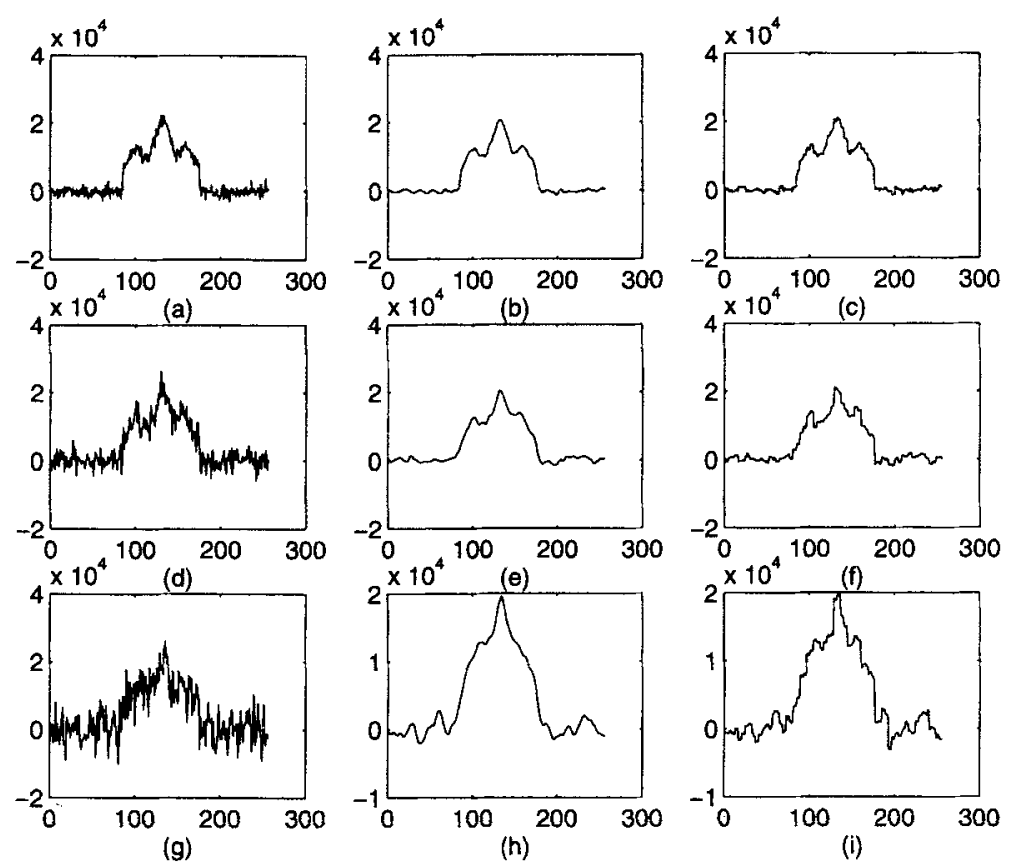

Figure 6. Noisy and estimated signals: (a), (d), (g) - noisy signals at 15,10 and $5 \mathrm{db}$ respectively; (b), (e), (h) - estimated signal using Daubechies' wavelet; (c), (f), (i) - estimated signals using Haar wavelet. 


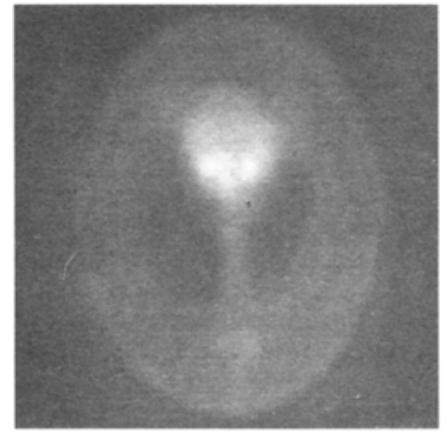

(a)

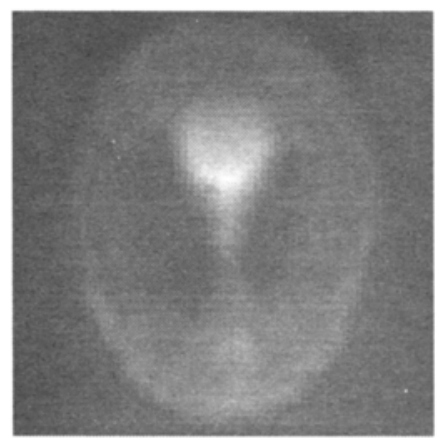

(d)

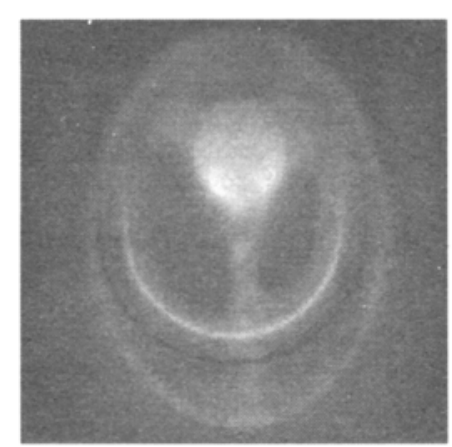

(b)

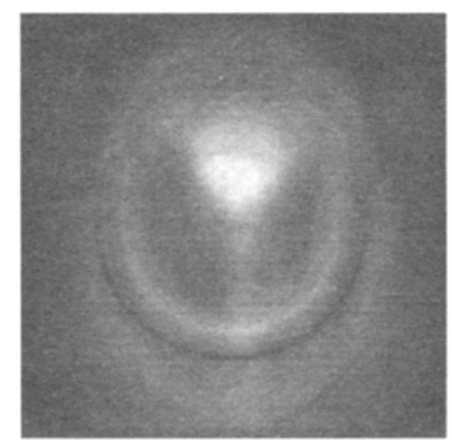

(e)

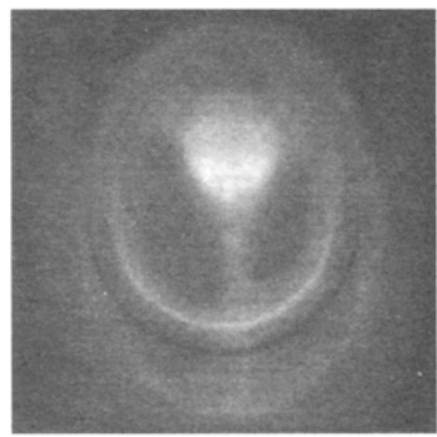

(c)

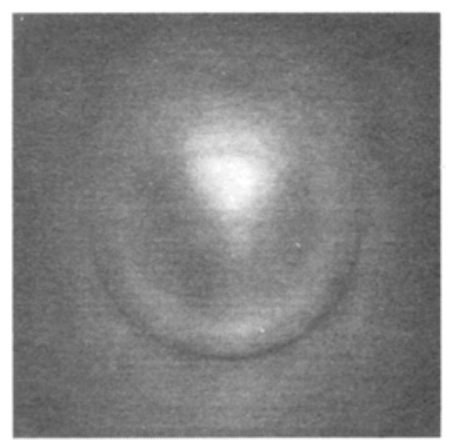

(f)

Figure 7. (d), (a) Reconstruction at two consecutive resolutions below the original respectively, at $15 \mathrm{~dB}$ SNR; (b), (c), (e), (f) - ROI reconstruction at high resolution with projections having $\mathrm{SNR}=20,15,10,5 \mathrm{db}$. respectively.

decorrelation increases with the regularity of the wavelet. For the Haar, $R=1$, while for the Daubechies' wavelet in our example, $R=5$. Figure 7 shows the result with different SNR ratios.

In figure $7 \mathrm{a}$ and $\mathrm{d}$, we show the multiresolution reconstruction at two different resolutions for an SNR of $15 \mathrm{~dB}$. Wiener filtering is easily applicable to multiresolution synthesis of a signal as it works directly in the scale space domain. Comparing with the original reconstruction from noiseless projections, we find the absence of fine scale features like edges from the low-resolution reconstruction.

Figure $8 b, c$, e and $f$ show the results for the ROI reconstruction. Within the ROI, the reconstruction is limited by the estimation procedure. The strength of the algorithm presented here (should be compared with (Bhatia \& Willsky 1996)) lies in the fact that localisation of the Radon transform and optimal estimation can be combined, using essentially the same property of wavelets, namely their regularity. The method presented here requires no simplifications in the estimation procedure. Also, the estimation being a scalar multiplication is not computationally demanding. 
(a)
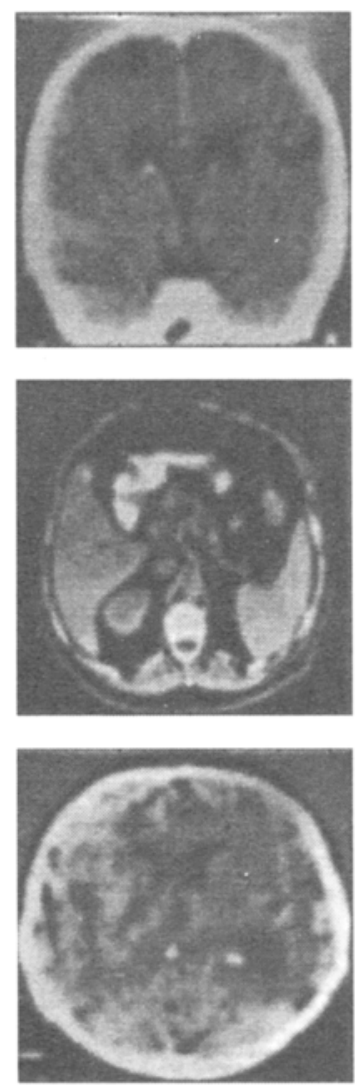

(b)
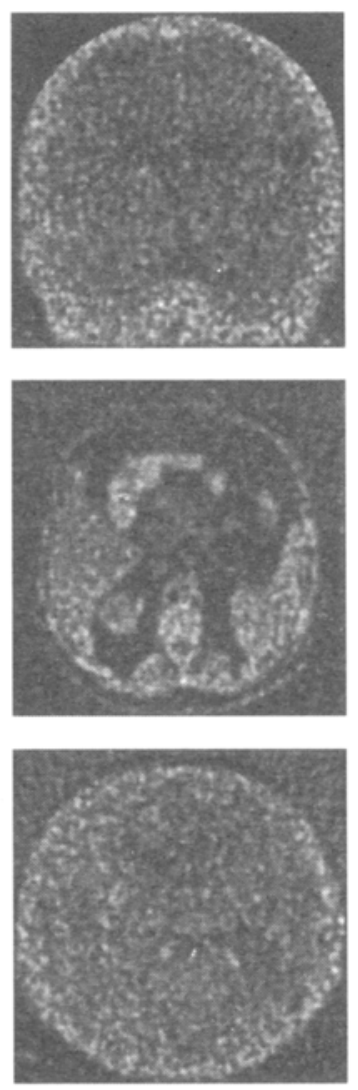

(c)
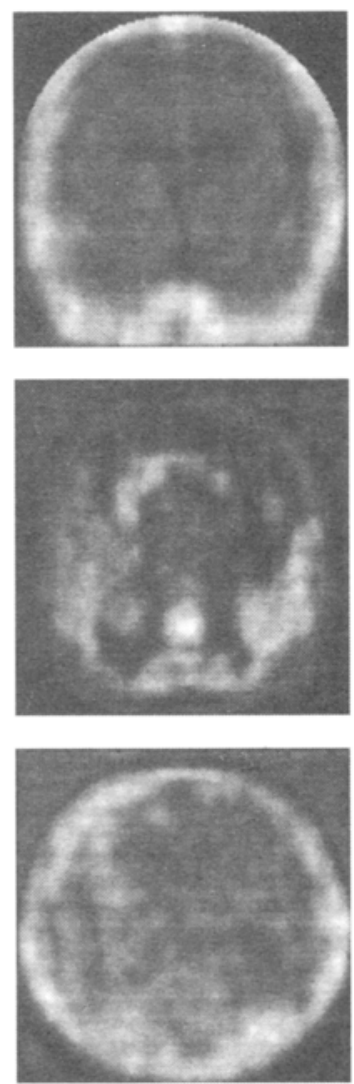

Figure 8. Reconstruction of the images from noisy projections at $15 \mathrm{~dB}$ SNR. The columns $(\mathbf{a}, \mathbf{b}, \mathbf{c})$ show the original image, noisy image and noise removed image respectively. The rows correspond to images $A, B, C$ respectively.

\subsection{How good is the $1 / f$ model?}

The entire algorithm described thus far hinges on the assumption that the projection of the image at each angle is a fractal signal. To test our assumption, we considered a few actual medical images obtained from medical texts. Here we show the results on three of the images we collected (figure 8). We refer to the images as A, B and C.

(A) CT scan of the large frontal lobe.

(B) Frontal CT scan in the investigation of the cortical areas (frontal, temporal, parietal), sellar, parasellar and third ventricle region.

(C) abdominal scan.

Figure 9 shows the linear trend in the detail coefficients across scales. The next two figures show the noise removal and reconstruction using this fractal model. The results obtained are certainly encouraging and strongly proves the fact that fractal model for medical image projections is a viable assumption. 


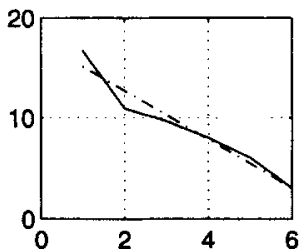

(a)

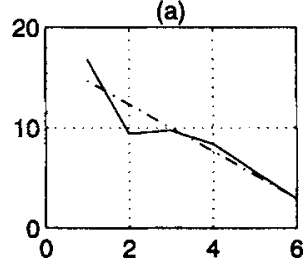

(d)

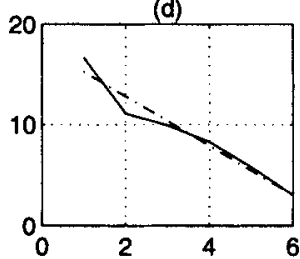

(g)

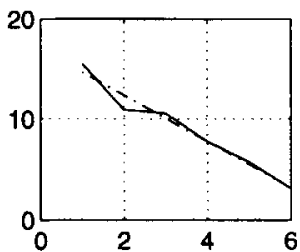

(b)

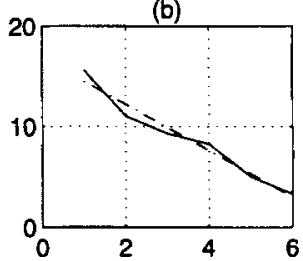

(e)

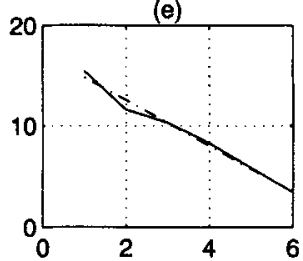

(h)

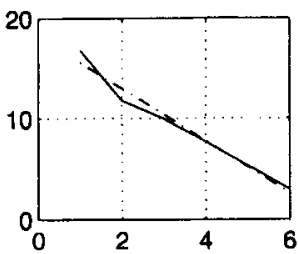

(c)

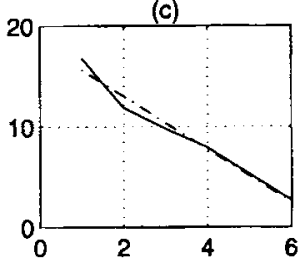

(f)

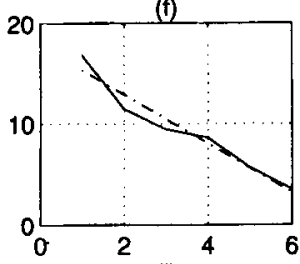

(i)

Figure 9. Each vertical column shows the straight line fit on three typical projections of images A, B, C. Column 1 corresponds to image A and so on. (- actual variance of the detail coefficients against scale; --- straight line fit.)

\section{Conclusion}

In this paper, we have shown how multiresolution and ROI reconstruction can be obtained when projections are noisy. For this purpose, we have assumed a fractal model for the projections and filtered the noise by using a Wiener filter. This filtering gives a multiscale output as it is done on the detailed coefficients obtained on performing a wavelet decomposition on the projections. These detailed coefficients are localised and hence we have used this property for ROI reconstruction.

\section{References}

Aldroubi A 1996 The wavelet transform: a surfing guide. In Wavelets in medicine and biology (Boca Raton, FL: CRC Press)

Bhatia M, Willsky A S 1996 A wavelet based method for multiscale tomographic reconstruction. IEEE Trans. Med. Imaging 15: 92-101

Barman K 1997 Multiscale processing of $1 / f$ signals. M E thesis. Electrical Engineering Department, Indian Institute of Science, Bangalore

Chui C K 1992 An introduction to wavelets (Boston, MA: Academic Press)

Daubechies I 1988 Orthonormal bases of compactly supported wavelets. Commun. Pure Appl. Math. 41: 909-996

Daubechies I 1992 Ten lectures on wavelets (Philadelphia: SIAM)

Delany A, Bresler Y 1995 Multiresolution tomographic reconstruction using wavelets. IEEE Trans. Image Process. 4: 799-813 
Kak A C, Roberts B A 1986 Reconstruction from projections. In Handbook of pattern recognition and image processing (Orlando, FL: Academic Press)

Masry E 1993 The wavelet transform of stochastic processes with stationary increments and its applications to fractional Brownian motion. IEEE Trans. Inf. Theory 39: 260-265

Natterer F 1986 The mathematics of computerized tomography (New York: John Wiley and Sons)

Olsen T 1996 Optimal time-frequency projection for localised tomography. In Wavelets in medicine and biology (Boca Raton, FL: CRC Press)

Olsen T, DeStefano J 1994 Wavelet localisation of the radon transform. IEEE Trans. Signal Processing 42: 2055-2067

Wornell G W 1993 Wavelet-based representation of $1 / f$ family of fractal processes. Proc. IEEE 81: $1428-1450$

Wornell G W, Oppenheim A V 1992 Estimation of fractal signals from noisy measurements using wavelets. IEEE Trans. Signal Process. 40: 611-625 